\title{
Les hôpitaux acteurs de développement
}

\author{
Certains hôpitaux suisses établissent des projets de coopération avec des hôpitaux \\ dans des pays en développement. Ce domaine revêt une importance particulière car \\ les hôpitaux possèdent une expertise et des moyens qui peuvent être utiles pour \\ développer les capacités dans les pays en voie de développement.
}

\section{Didier Wernli, Gabrielle Landry-Chappuis, Slim Slama, Louis Loutan}

Service de médecine internationale et humanitaire, Hôpitaux Universitaires de Genève; Faculté de médecine, Université de Genève

\section{Correspondance:}

Prof. Dr Louis Loutan

Service de médecine internationale et humanitaire Hôpitaux Universitaires de Genève

4, rue Gabrielle-Perret-Gentil CH-1211 Genève 14

Tél. 0223729613

louis.loutan@hcuge.ch
Depuis de nombreuses années, certains hôpitaux suisses établissent des projets de coopération avec des hôpitaux dans des pays en développement. Ces collaborations sont souvent le fruit de rencontres entre professionnels - médecins, infirmières ou administrateurs - qui décident de monter un projet commun. Il peut notamment s'agir d'activités de formation, de l'introduction de nouvelles méthodes de gestion, du développement, d'échanges professionnelles régulières d'une équipe chirurgicale pour aller opérer dans un hôpital, ou encore du déplacement de toute autre collaboration destinée à renforcer les capacités de l'institution partenaire. Ces diverses initiatives sont très riches tant au niveau professionnel qu'humain et, petit à petit, tout un réseau de contacts et de compétences se développe. La télémédecine et des moyens de communication via internet peuvent largement faciliter ces partenariats.

A l'heure qu'il est, la contribution des hôpitaux suisses à l'effort de coopération au développement est très mal documentée et peu reconnue. Or cette dernière est non négligeable. Ce domaine revêt une importance particulière car les hôpitaux constituent un des principaux acteurs du système de santé. Ils possèdent notamment une expertise et des moyens qui peuvent être utiles pour développer les capacités dans les pays en voie de développement. Aussi nous est-il apparu important de répertorier ces partenariats au niveau Suisse. Ce travail est le résultat d'une collaboration entre l'organisation faîtière des hôpitaux et cliniques suisses, l'association $\mathrm{H}+$ et le Service de médecine internationale et humanitaire (SMIH) des Hôpitaux Universitaires de Genève (HUG).

\section{Méthode}

Il s'agit d'une enquête menée sur la base d'un questionnaire électronique, réalisée en deux temps, du 16 octobre 2007 au 31 mars 2009. Tous les hôpitaux et cliniques de Suisse ont été contactés en utilisant les adresses mises à disposition par l'association $\mathrm{H}+$.

Un premier questionnaire électronique, cosigné par l'association $\mathrm{H}+$ et le $\mathrm{SMIH}$, a été envoyé à plus de 200 hôpitaux et cliniques suisses, suivi d'un rappel deux mois plus tard. Le document était rédigé en allemand, en français et en italien. Le questionnaire com-

\section{Résumé}

De nombreux hôpitaux et cliniques suisses possèdent des projets de coopération au développement. Cependant, leur contribution à l'effort de coopération au développement est très mal documentée et peu reconnue. Cet article présente les résultats d'une enquête visant à répertorier les projets inter-hospitaliers dans les hôpitaux et cliniques suisses. Les résultats montrent une grande diversité de projets de partenariats, dont la majorité contribue à développer les capacités professionnelles et institutionnelles locales. Finalement, l'article propose des recommandations pour améliorer l'intégration des projets interhospitaliers dans l'aide au développement à l'image de ce qui se fait dans certains pays européens.

prenait cinq questions visant à établir la présence et le nombre de projets de coopération en cours ou programmés, l'existence d'une stratégie institutionnelle de coopération internationale et la mise à disposition de fonds spécifiques à cet effet.

\section{Ces diverses initiatives sont très riches tant au niveau professionnel qu'humain}

A l'issue de cette première partie, les institutions rapportant des projets ont reçu un deuxième questionnaire, suivi d'un rappel. Celui-ci comportait neuf questions visant à caractériser les projets en termes de secteurs d'activités (médical, logistique/gestion ou infirmier), spécialités médicales selon la liste FMH (chirurgie, médecine interne, pédiatrie, etc.), types d'activités (enseignement/formation, activités clini- 
ques, recherche, etc.), répartition géographique et sources de financement. Pour augmenter le taux de réponse les personnes de référence ont été contactées pour les engager à répondre au questionnaire.

\section{Résultats}

Lors de la première partie de l'enquête, 62 institutions hospitalières ont répondu au questionnaire en ligne. Sur ces 62 hôpitaux et cliniques, 30 mènent des projets de coopération totalisant 93 projets. Parmi ces projets, 58 proviennent de quatre institutions: HUG, Centre Hospitalier Universitaire Vaudois (CHUV), Hôpital Riviera à Vevey et Kantonsspital de SaintGall. 43\% des institutions (13/30), comptabilisant 55 projets, possèdent une stratégie institutionnelle de coopération internationale. Concernant le financement, $27 \%$ des hôpitaux et cliniques (8/30) ont un fonds spécifique interne pour les projets humanitaires ou de coopération. Cinq hôpitaux et cliniques romandes (CHUV, Clinique Belmont, Clinique des Grangettes, Hôpital Riviera et HUG) possèdent à la fois une stratégie institutionnelle et un mécanisme de financement spécifique.

Le second questionnaire, envoyé aux 30 hôpitaux ayant déclaré des projets, a permis de recevoir des précisions concernant 35 projets. A la suite d'appels téléphoniques réalisés début 2009 aux institutions concernées, 11 projets supplémentaires ont été identifiés. Au total, ce sont donc 46 projets, équivalant à $49 \%(46 / 93)$ du total des projets annoncés dans la première partie, qui ont été détaillés grâce à l'enquête.

Au niveau géographique (fig. 1), il existe une prédominance de projets dans des pays d'Afrique (31/46) suivi de projets en Europe (23/46) majoritairement en Europe de l'est (22/23), et enfin, dans une moindre

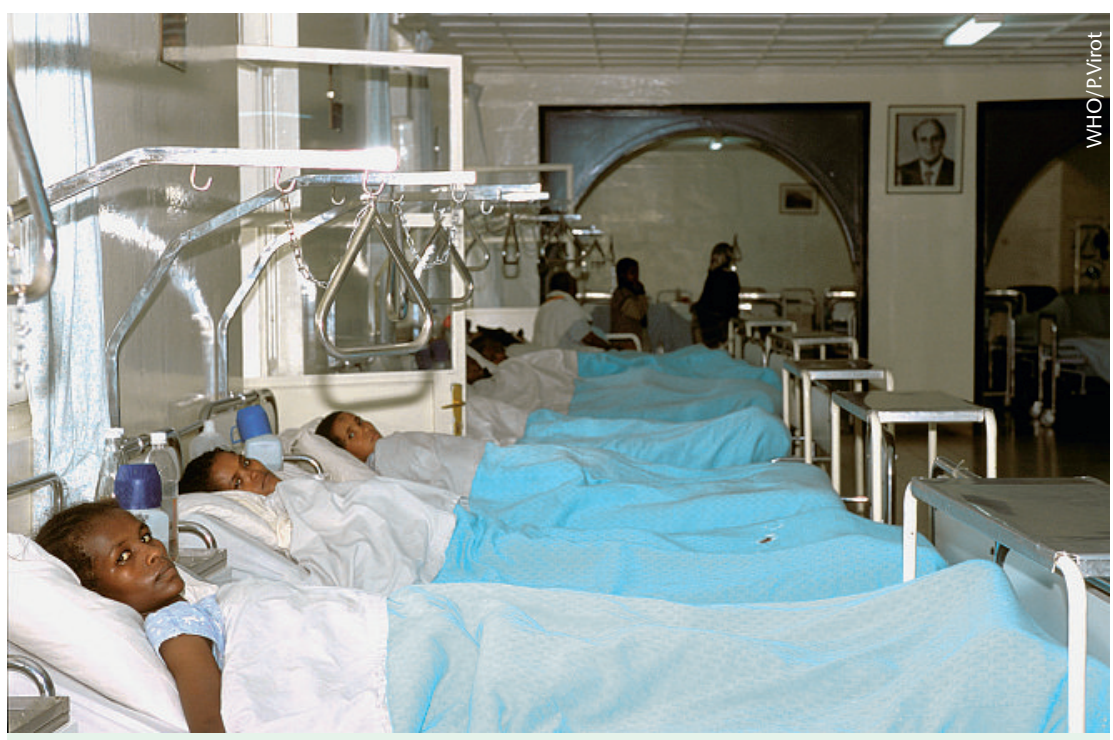

Depuis de nombreuses années, certains hôpitaux suisses établissent des projets de coopération avec des hôpitaux dans des pays en développement.
Figure 1

Répartition des projets par régions géographiques.

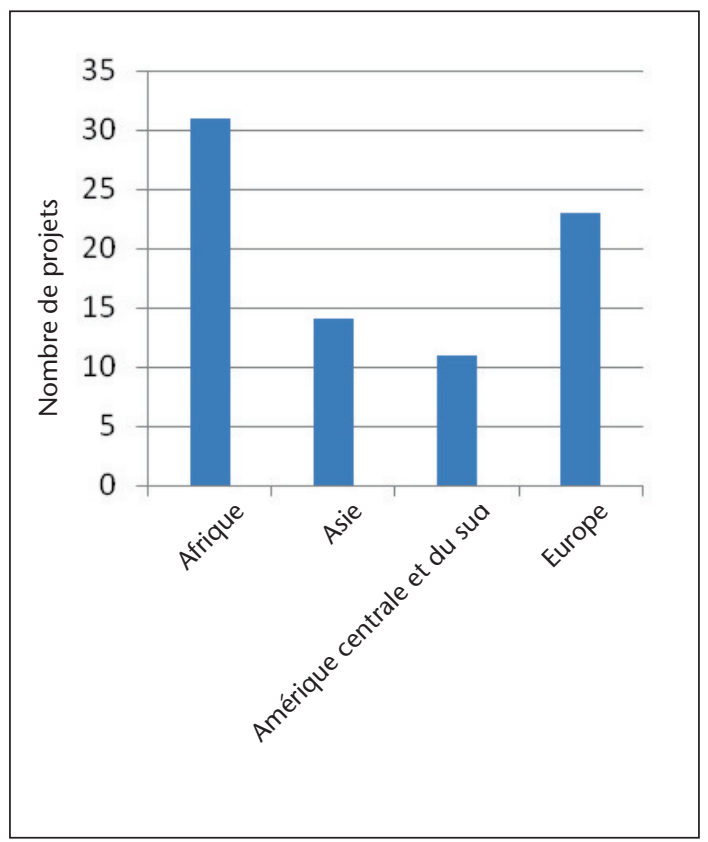

mesure, en Asie (14/46) et Amérique latine (11/46). Un petit nombre de projets (7/46) concernent plusieurs pays.

Parmi les projets répertoriés, une majorité (91\%) possède une composante médicale $(42 / 46)$. Les projets en soins infirmiers représentent plus d'un tiers du total $(17 / 46)$. Au total $24 \%(11 / 46)$ portent sur un appui logistique, technique ou administratif.

L'ensemble des projets couvrent un large spectre de spécialités médicales (fig. 2). Parmi celles-ci, la chirurgie et l'anesthésiologie représentent $46 \%$ des projets $(21 / 46)$. 39\% des projets relèvent de la médecine interne et de ses spécialités (18/46) et $17 \%$ concernent le domaine de la santé mère-enfant (gynécologie-obstétrique: $\mathrm{n}=3$ et pédiatrie: $\mathrm{n}=5$ ). Finalement, 37\% des projets touchent à plus d'une spécialité (17/46).

La plupart des projets englobent plusieurs types d'activités (fig. 3). Environ $80 \%$ des projets comprennent un volet d'enseignement et/ou de formation (36/46) dont 5 sont uniquement de l'enseignement et/ou formation. Un peu plus de la moitié des projets (52\%) présentent une composante clinique ou de soins (24/46). Seul un projet compte une activité clinique ou de soins uniquement. Les projets de recherche s'élèvent à 37\% (17/46). Trois projets sont uniquement dédiés à la recherche. Les dons de matériel se retrouvent dans un peu moins de $30 \%$ des projets (13/46). Dans la plupart des cas, ces dons de matériel sont associés à d'autres types d'activités (9/13).

Finalement, en termes de financement, $63 \%$ des projets (29/46) sont financés par des fonds propres, en totalité (12/29) ou partiellement (17/29). Des fonds externes appuient $74 \%$ des projets $(34 / 46)$, en totalité 
Figure 2

Répartition des projets selon spécialités médicales (liste FMH).

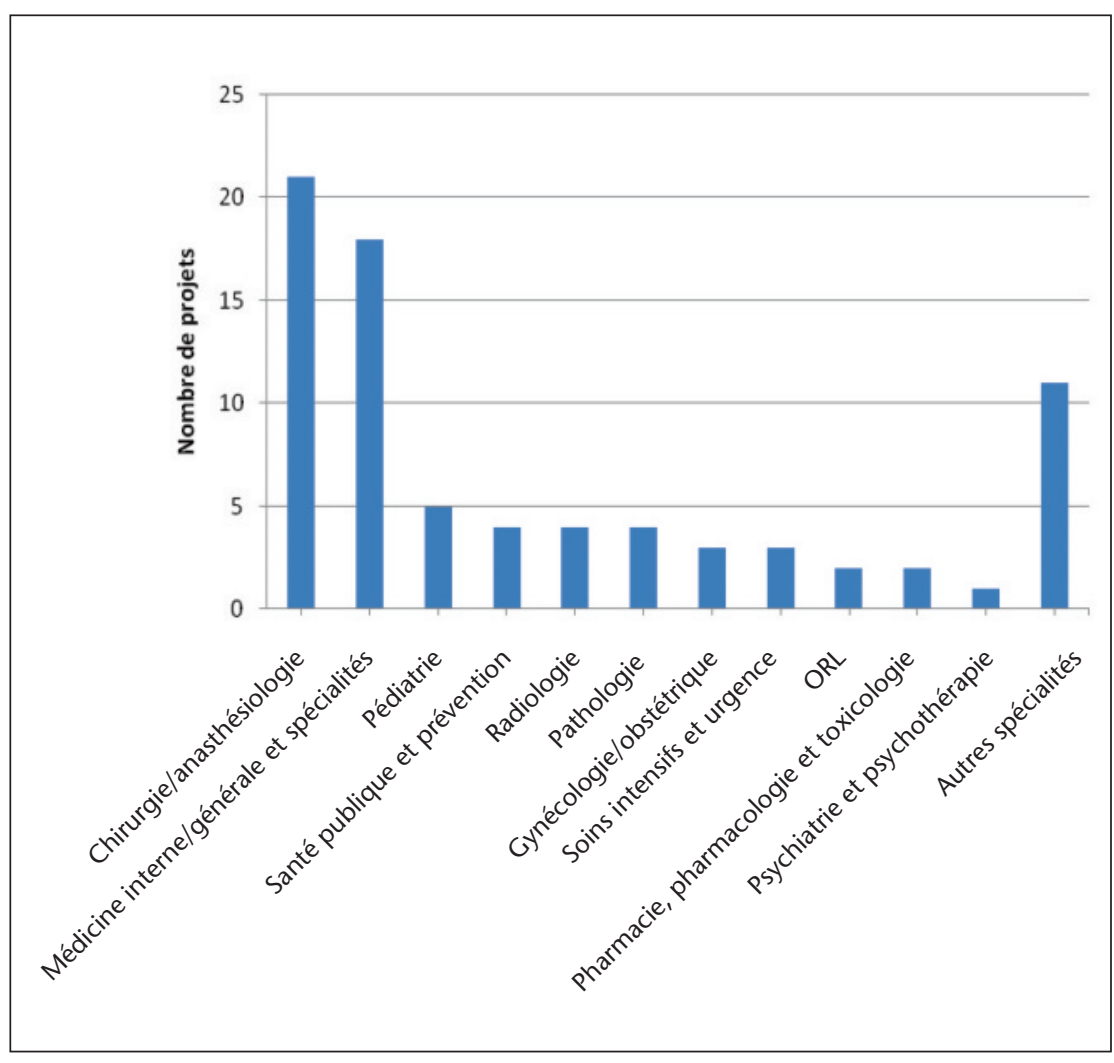

Figure 3

Répartition des projets par types d'activités. (En rouge apparaissent les projets qui se concentrent uniquement sur leur catégorie).

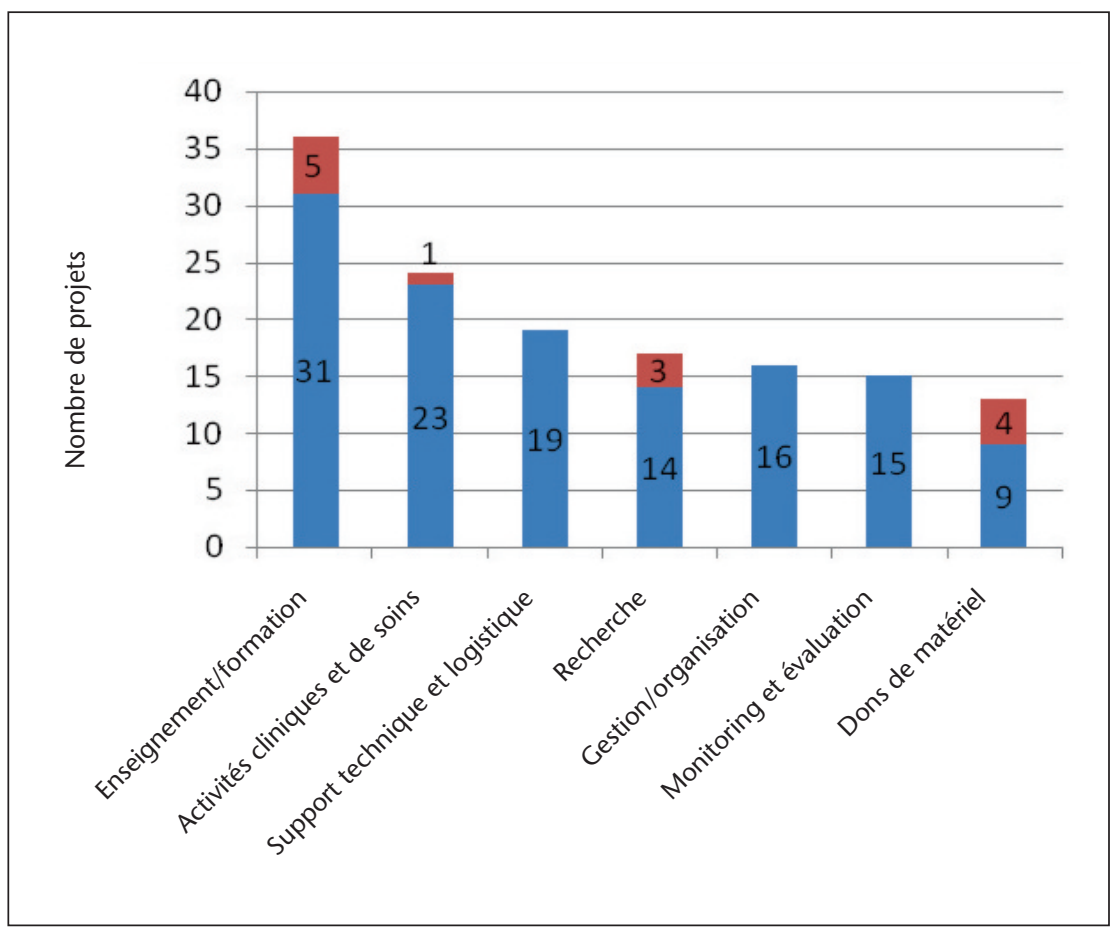

du financement (17/46) ou partiellement (17/46). La Direction de la Coopération et du Développement (DDC) finance $15 \%$ des projets (7/46), partiellement (6) ou en totalité (1).

\section{Discussion}

Cette enquête met en évidence le nombre et la diversité des projets de coopération internationale menés par 30 hôpitaux et cliniques de Suisse. Très probablement ces résultats sous-estiment le nombre réel des collaborations internationales avec des pays en développement. En envoyant le questionnaire à la direction des hôpitaux et cliniques de Suisse, il est probable que des projets non répertoriés comme tels au niveau institutionnel n'aient pas été rapportés. Malgré cette limitation, cette première évaluation permet de formuler un certain nombre d'observations.

Il faut tout d'abord relever que ces projets sont aussi bien le fait d'hôpitaux publics universitaires ou régionaux que de cliniques privées. Les résultats montrent qu'il existe, par ailleurs, une grande variété de projets avec des activités allant de la santé maternelle et infantile à la chirurgie, en passant par la santé mentale et la radiologie. Si la chirurgie et les différentes branches de la médecine sont les plus représentées, des secteurs moins classiques comme la pharmacologie, l'ORL, les soins intensifs ou encore la télémédecine sont présents. La majorité des projets ont plusieurs composantes et ne se limitent certainement pas à des prestations purement cliniques chirurgicales ou médicales. Il semble que, le plus souvent, on est loin du modèle de l'équipe médicale ou chirurgicale qui fait des visites ponctuelles pour effectuer des interventions que les équipes locales ne sont pas à même de mener, faute de moyens et de compétences techniques nécessaires [1]. Les dons de matériel non accompagnés d'autres activités ne représentent que $9 \%$

\section{Sur ces 62 hôpitaux et cliniques, 30 menaient des projets de coopé- ration totalisant 93 projets}

(4/46) des projets. Plus de trois quarts des projets (36/46) apportent une dimension de formation ou d'enseignement, d'appui technique ou logistique ou encore de soutien en gestion et en organisation. Clairement, ces partenariats contribuent à développer les capacités professionnelles voire institutionnelles locales. L'étude met donc en lumière le tournant qui s'est opéré dans l'aide depuis les années 1980 où le modèle se résumait à financer des investissements et à mettre à disposition des ressources humaines [1] .

Autre fait important, on note que les projets recensés dépassent l'élan de générosité humanitaire pour s'inscrire dans $43 \%$ des cas dans une stratégie institu- 
tionnelle. C'est dire que cet engagement commence à se structurer. Les HUG constituent un bon exemple de ce phénomène. En effet, le développement des activités internationales figurent en bonne place dans le plan stratégique. Une quote-part est notamment prélevée sur l'ensemble des consultations privées pour alimenter un fonds permettant de soutenir des projets et d'en assurer une certaine continuité. Cette volonté de soutenir la coopération s'est cristallisée dans la création d'un service de médecine humanitaire et internationale dont l'une des missions est de renforcer les activités humanitaires et de coopération internationale des HUG.

Le financement des projets tend également à se structurer et à se diversifier. Un quart des hôpitaux (27\%) possèdent des fonds propres pour leurs projets de coopération (63\% des projets). En outre des sources
- Développer les compétences culturelles personnelles, professionnelles et institutionnelles;

- Motiver les équipes soignantes dans un projet pouvant renforcer la cohésion et l'engagement du service;

- Mieux comprendre des nouveaux enjeux globaux de la santé et de la médecine.

Au-delà du cadre hospitalier, ces projets de coopération sont aussi une valeur ajoutée dans la formation des futurs praticiens qui peuvent être impliqués, également, une fois installés, dans des partenariats internationaux.

Dans les faits, le modèle de l'aide à court terme fait place à celui du développement de partenariats institutionnels durables, dans lequel les partenaires contribuent et bénéficient mutuellement de ces

\section{Le modèle de l'aide à court terme fait place à celui du développement de partenariats institutionnels durables}

externes sont mobilisées dans $74 \%$ des projets. Finalement, les données indiquent que la DDC n'est impliquée que dans $15 \%$ des partenariats hospitaliers.

Pour finir, il faut mentionner que ce premier sondage n'avait pas l'ambition d'évaluer l'impact et l'efficacité de ces interventions, ni de s'enquérir des règles et processus suivis dans ces partenariats. Toutefois, il donne un premier aperçu des partenariats hospitaliers avec des pays en développement et l'engagement des hôpitaux et cliniques suisses dans la coopération au développement dans le domaine de la santé.

\section{Perspectives et recommandations}

Cette étude illustre l'engagement des institutions hospitalières à participer à l'effort de coopération suisse notamment par le renforcement des capacités des partenaires locaux. On ne manquera pas de relever, par ailleurs, que les bénéfices de tels liens ne se limitent pas aux récipiendaires de l'aide. En effet, ils permettent également aux institutions hospitalières de développer leurs réseaux de contacts et de promouvoir les compétences professionnelles [1]. Les bénéfices de ce type de coopération peuvent revêtir plusieurs formes:

- Elargir les compétences professionnelles en pratiquant dans un environnement moins technologique avec une grande diversité de pathologies;

- Améliorer les capacités d'adaptation et de gestion dans un contexte nouveau;

- Offrir des opportunités de recherche (études comparatives multicentriques, nouveaux domaines et dimensions de recherche);

- Renforcer les capacités de formation et d'enseignement (développement de curriculum, formations, échanges de professionnels ou d'étudiants); échanges. Ce phénomène s'inscrit dans un contexte où la notion de pays en développement tend à se modifier à mesure que certains pays connaissent une croissance rapide sur le plan économique et qu'ils améliorent leurs compétences et la qualité de leurs institutions. A l'avenir, on peut s'attendre à ce qu'une part grandissante de la formation avancée des médecins dans nos institutions se fasse dans les pays émergents comme l'Inde ou la Chine, où bien souvent cohabitent des institutions de haut niveau technologique avec des structures s'apparentant aux pays en

\section{Les partenaires contribuent et bénéficient mutuellement de ces échanges}

développement. C'est maintenant que ces partenariats interinstitutionnels doivent être établis. A ce propos, les nombreux professionnels médecins, infirmières ou techniciens travaillant dans nos hôpitaux ou exerçant en pratique privée, peuvent faciliter ces partenariats et jouer un rôle de tête de pont avec des institutions dans leur pays d'origine.

A mesure que les pays émergents poursuivent leur développement, les hôpitaux comme structure de soins, d'expertise, de recherche et de formation seront probablement amenés à jouer un rôle croissant dans la coopération. Au niveau des maladies chroniques, l'urbanisation rapide des pays en développement et le vieillissement de la population influencent largement les changements épidémiologiques vers des patholo- 
gies non infectieuses prédominantes en Suisse et en Europe. Cette progression des maladies chroniques ainsi que le développement de résistances dans le domaine des maladies infectieuses vont considérablement complexifier les activités diagnostiques et thérapeutiques, nécessitant une expertise médicale plus pointue dans les pays en développement. De ce fait, l'expertise disponible dans nos hôpitaux en matière de soins aigus ou chroniques, hospitaliers ou ambulatoires devient très utile une fois adaptée aux conditions locales.

Une meilleure définition du rôle des hôpitaux dans les systèmes de santé devient incontournable. Certains pays, à l'instar du Royaume-Uni, ont bien compris l'importance des partenariats hospitaliers et ont inscrit ceux-ci dans leur stratégie d'aide au développement [2-4]. A titre d'exemple, la plateforme International Health Links Centre réunit les expériences des différents acteurs britanniques impliqués dans des projets de coopération avec des pays en voie de développement [5]. Cette volonté d'impliquer les acteurs hospitaliers s'est traduite par la mise à disposition de fonds spécifiques favorisant la création de nouveaux liens. En Suisse, une prise de conscience commence à s'opérer. On notera à cet égard, la tenue, lors de la dernière édition du Geneva Health Forum en avril 2010, d'une session sur le thème des partenariats hospitaliers. Cette session a débouché sur la mise sur pied d'un groupe de réflexion réunissant des acteurs français, britanniques, camerounais et suisses.

En conclusion, dans le but de mieux valoriser la participation des institutions hospitalières dans le domaine de la coopération en Suisse, il existe un certain nombre de pistes à explorer:

- Recenser régulièrement les partenariats inter-hospitaliers;

- Créer une plateforme électronique permettant aux institutions intéressées de développer des synergies (partage d'information et d'expérience, identification rapide de l'expertise.);

- Evaluer l'impact des partenariats en adoptant une approche systématique basée notamment sur la Déclaration de Paris sur l'efficacité de l'aide [6];

- Etablir des codes de bonne pratique à l'image de ceux développés dans les partenariats de recherche (KFPE) [7];

- Mettre sur pied une structure multipartite associant divers acteurs de la coopération suisse et de la santé globale visant à entamer une réflexion sur les manières de mieux intégrer les projets de coopération des institutions hospitalières suisses dans l'effort de coopération suisse.

\section{Remerciements}

Nous remercions l'association $\mathrm{H}+$ pour avoir soutenu et facilité ce sondage effectué auprès des hôpitaux et cliniques suisses membres de l'association.

\section{Références}

1 de Roodenbeke E. Une voie d'avenir pour la cooperation: le partenariat hospitalier. Santé. 1994;4:105-9.

2 Crisp N. Global Health Partnerships. The UK Contribution to Health in Developing Countries. London: Department of Health; 2007.

3 Department of Health. Health is global: proposals for a UK government-wide strategy. London; 2007.

4 Department of Health. The Framework for NHS Involvement in International Development. 2010.

5 www.ihlc.org.uk/

6 Organisation de Coopération et de Développement Economiques. Déclaration de Paris sur l'efficacité de l'aide. 2005.

7 Commission pour le partenariat scientifique avec les pays en développement (KFPE). Guidelines for Research in Partnership with Developing Countries. 1998. 